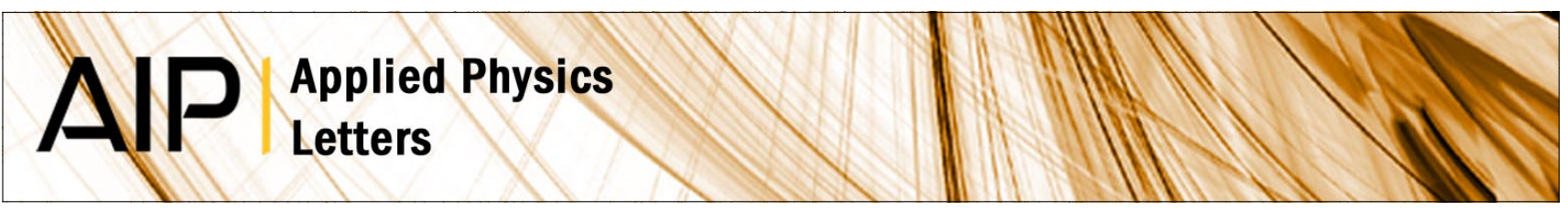

\title{
Size effect on the coalescence-induced self-propelled droplet
}

Feng-Chao Wang, Fuqian Yang, and Ya-Pu Zhao

Citation: Appl. Phys. Lett. 98, 053112 (2011); doi: 10.1063/1.3553782

View online: http://dx.doi.org/10.1063/1.3553782

View Table of Contents: http://apl.aip.org/resource/1/APPLAB/v98/i5

Published by the American Institute of Physics.

\section{Related Articles}

Surface charge density model for predicting the permittivity of liquid mixtures and composites materials J. Appl. Phys. 111, 064101 (2012)

Energy dissipation in non-isothermal molecular dynamics simulations of confined liquids under shear J. Chem. Phys. 135, 134708 (2011)

Extension of the Steele 10-4-3 potential for adsorption calculations in cylindrical, spherical, and other pore geometries

J. Chem. Phys. 135, 084703 (2011)

Melting of iron at the Earth's core conditions by molecular dynamics simulation

AIP Advances 1, 032122 (2011)

Equilibrium structure of the multi-component screened charged hard-sphere fluid

J. Chem. Phys. 135, 014504 (2011)

\section{Additional information on Appl. Phys. Lett.}

Journal Homepage: http://apl.aip.org/

Journal Information: http://apl.aip.org/about/about_the_journal

Top downloads: http://apl.aip.org/features/most_downloaded

Information for Authors: http://apl.aip.org/authors

\section{ADVERTISEMENT}

\section{NEW!}

iPeerReview

AIP's Newest App

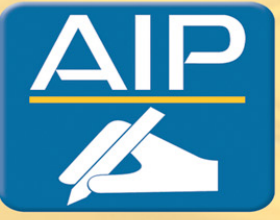

Authors...

Reviewers...

Check the status of

submitted papers remotely!

ADPublishing 


\title{
Size effect on the coalescence-induced self-propelled droplet
}

\author{
Feng-Chao Wang, ${ }^{1}$ Fuqian Yang, ${ }^{2, a)}$ and Ya-Pu Zhao ${ }^{1, a)}$ \\ ${ }^{1}$ State Key Laboratory of Nonlinear Mechanics, Institute of Mechanics, Chinese Academy of Sciences, \\ Beijing 100190, People's Republic of China \\ ${ }^{2}$ Department of Chemical and Materials Engineering, University of Kentucky, Lexington, \\ Kentucky 40506, USA
}

(Received 8 November 2010; accepted 21 January 2011; published online 4 February 2011)

\begin{abstract}
An analysis based on the energy conservation is presented for the self-propelled droplet during coalescence of two droplets of the same size over a superhydrophobic rough surface. The self-propelled behavior occurs only for the coalescence of droplets with a certain range of radius. An analytical relation is established among the coalescence-induced velocity, surface energy, viscous dissipation, and droplet size if gravity is negligible. The coalescence-induced velocity increases with increasing droplet size to a maximum and then decreases with the size, which is in good accord with the experimental observation reported in the literature. () 2011 American Institute of Physics. [doi:10.1063/1.3553782]
\end{abstract}

Depending on the bonding energy between liquid molecules and surface atoms/molecules of a solid, a solid surface can be wettable or nonwettable. Through chemical treatment, such as the grafting of hydrogenated or fluorinated chlorosilanes onto the $\mathrm{Si}-\mathrm{OH}$ groups of a glass surface, ${ }^{1}$ one can control the wettability of a solid surface. In general, wettability determines the detachment and wetting of droplets or bubbles on a solid surface, which is of practical importance for many applications in self-cleaning, microfluidics, lab-on-a-chip devices, and liquid-based cooling of microelectronics.

Recently, there has been a great interest in developing techniques to achieve the transition of the Wenzel state ${ }^{2}$ to the Cassie-Baxter state ${ }^{3}$ for separating liquid from solid surfaces, which include vibration-induced dewetting ${ }^{4}$ and impulse heating. ${ }^{5}$ All these works involved the use of external energy, such as mechanical energy and electric energy, to overcome the energy barrier between these two states. To reduce the energy barrier and the wetting of liquid, hydrophobic or superhydrophobic surfaces are desired and different methods have been developed to form synthetic superhydrophobic surfaces. The use of superhydrophobic surfaces in the dropwise condensation has led to the observation of the rapid motion of liquid droplet driven by the coalescence. ${ }^{6-9}$ Such a phenomenon is believed to be powered by the surface energy released during the drop coalescence. Boreyko and Chen ${ }^{6}$ did a simple scaling analysis by assuming that all the released surface energy is converted to kinetic energy of the merged droplet. However, a quantitative understanding of the relationship between energy and kinetics has not been resulted.

In this work, a quantitative approach is proposed, whereby the kinetics of the coalescent droplet is governed by an equilibrium balance of surface energy, kinetic energy, and the viscous dissipation energy. The gravitational-potential energy can be neglected in the analysis since the scale of the droplets is much smaller than the capillary length

\footnotetext{
a) Authors to whom correspondence should be addressed. Electronic addresses: fyang0@engr.uky.edu and yzhao@imech.ac.cn.
}

$l_{c}=\sqrt{\gamma_{l v} / \rho g}(2.7 \mathrm{~mm}$ for water), in which $g$ is the gravitational acceleration, $\rho$ is the density of the liquid, and $\gamma_{l v}$ is the surface tension of the liquid-vapor interface. The interaction energy between two droplets with radius of $r_{1}$ and $r_{2}$, respectively, can be calculated as $W=-A_{H} r_{1} r_{2} / 6 D\left(r_{1}+r_{2}\right),{ }^{\text {ro }}$ where $A_{H}$ is the Hamaker constant and $D$ is the separation distance between the two droplets. The interaction energy is about six to seven orders of magnitude less than the surface energy and the viscous dissipation energy. Thus, the interaction energy between two droplets is negligible in the analysis.

The concept developed will be for the coalescence of two quasistationary liquid droplets of the same size on a superhydrophobic rough surface. The method can be applied with some modifications to more complicated situations involving the coalescence of multiple droplets. Consider two droplets of the same radius $r$, which coalesce to form a new droplet of radius $R$, as shown in Fig. 1. Under the assumption of the absence of gravity (the typical Bond number Bo $\left.=\rho g r^{2} / \gamma_{l v} \ll 1\right)$, the equilibrium shape of a droplet is a spherical cap with an apparent contact angle $\theta$.

The surface energy of each stationary droplet on a hydrophobic rough surface is given by

$$
E_{S}=\gamma_{l v} A_{l v}+\gamma_{s l} A_{s l}+\gamma_{s v} A_{s v},
$$

where $A$ is the interfacial area and the subscripts $s, l$, and $v$ denote the solid, liquid, and vapor, respectively. For a rough

\section{Wenzel state Cassie-Baxter state}

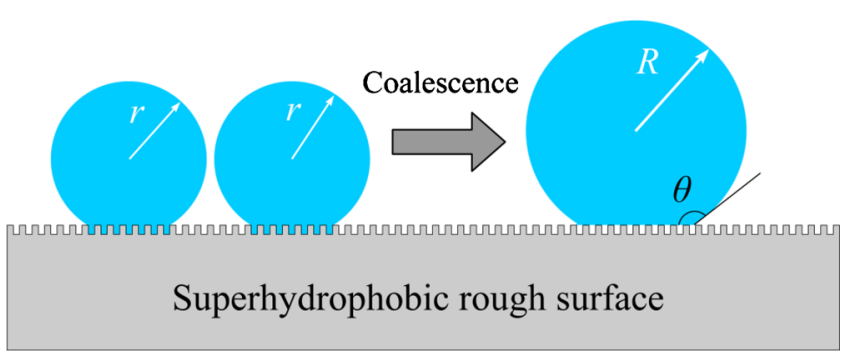

FIG. 1. (Color online) Schematic of the coalescence of two droplets to one big droplet on a superhydrophobic rough surface. The transition of the Wenzel state to the Cassie-Baxter state is shown. 
surface, the surface energy can be written as ${ }^{11}$

$$
E_{S}=\gamma_{l v} \pi r^{2}\left[2-2 \cos \theta-\Psi(f) \sin ^{2} \theta\right]+\gamma_{s v} A_{\mathrm{tot}},
$$

in which $A_{\text {tot }}$ is the total area of the solid surface and

$$
\Psi(f)=r_{f} f \cos \theta_{Y}+f-1,
$$

where $r_{f}$ is the roughness ratio of the wet surface area, $f$ is the fraction of the solid surface area wetted by the liquid, and $\theta_{Y}$ is the Young contact angle as defined for an ideal surface. Before coalescence, the droplets are in the Wenzel state, which means $f=1$. A transition from the Wenzel to the Cassie-Baxter state due to the coalescence can be observed. ${ }^{6}$ Thus, the surface energy of the droplet after coalescence has a similar expression as Eq. (2), while the parameters $r_{f}$ and $f$ should be changed. $\Psi_{W}(f)$ and $\Psi_{C}(f)$ would be used to distinguish the coefficients for the Wenzel and the CassieBaxter state, respectively.

During the coalescence, there exists the energy dissipation due to the flow and mergence of the liquid droplets against viscosity. Approximately, the viscous dissipation energy for each droplet can be estimated as ${ }^{12}$

$$
E_{\mathrm{vis}}=\int_{0}^{\tau} \int_{\Omega^{\prime}} \Phi \mathrm{d} \Omega^{\prime} \mathrm{d} t \approx \Phi \Omega \tau,
$$

in which $\Phi$ is the dissipation function

$$
\Phi=\frac{\mu}{2}\left(\frac{\partial v_{i}}{\partial x_{j}}+\frac{\partial v_{j}}{\partial x_{i}}\right)^{2} \approx 12 \mu\left(\frac{U}{r}\right)^{2},
$$

$\Omega$ is the volume of each droplet, $\mu$ is the viscosity of the liquid, and the characteristic capillary time scale $\tau$ is given by

$$
\tau \approx \sqrt{\rho r^{3} / \gamma_{l v}} .
$$

As the coalescence starts, the capillary pressure inside the droplet $\left(\Delta p=2 \gamma_{l v} / r\right)$ will accelerate the droplet along the horizontal direction. ${ }^{13}$ Thus, the average velocity $U$ of each droplet can be obtained as

$$
U \approx \tau \cdot \Delta p \cdot \pi r^{2} \frac{1}{4 \rho \pi r^{3} / 3}=\frac{3}{2} \sqrt{\frac{\gamma_{l v}}{\rho r}} .
$$

Combining Eqs. (4)-(7) yields

$$
E_{\mathrm{vis}} \approx 36 \pi \mu \sqrt{\frac{\gamma_{l v} r^{3}}{\rho}} .
$$

The total energy of the system after coalescence consists of surface energy and kinetic energy of the coalescent droplet. Then, balancing the change in the total energy of the system due to the coalescence and introducing the mass conservation, i.e., $R^{3}=2 r^{3}$, one obtains the kinetic energy of the coalescent droplet as

$$
\begin{aligned}
E_{K}= & \gamma_{l v} \pi r^{2}\left\{\left(2-2^{2 / 3}\right)(2-2 \cos \theta)+\left[2^{2 / 3} \Psi_{C}(f)\right.\right. \\
& \left.\left.-2 \Psi_{W}(f)\right] \sin ^{2} \theta\right\}-64 \pi \mu \sqrt{\frac{\gamma_{l v} r^{3}}{\rho}}
\end{aligned}
$$

which depends on the size of the starting droplets and the wetting condition of the rough surface. Some portions of the surface energy released upon the coalescence will be used to balance the viscous dissipation energy. To have the coalescence-induced self-propelled droplet, it requires $E_{K}$ $>0$. It can be seen from Eq. (9) that the coalescence-induced

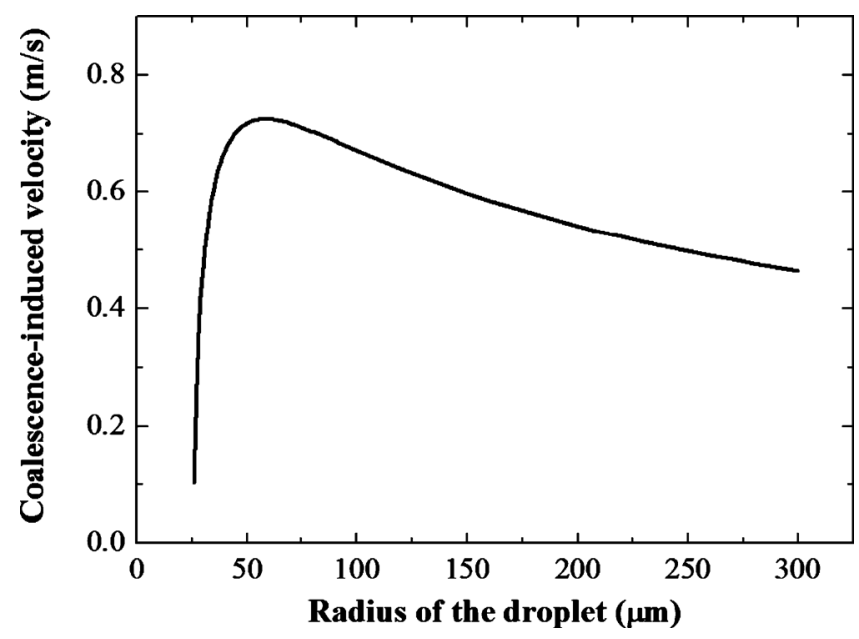

FIG. 2. Dependence of the coalescence-induced velocity on the radius of the droplet (the parameters used in the calculation are $\theta=170^{\circ}, \theta_{Y}=160^{\circ}, r_{f W}$ $=1.5, f_{W}=1.0, r_{f C}=1.0$, and $f_{C}=0.5$; the properties of water at $20{ }^{\circ} \mathrm{C}$ are used, such as $\rho=998.23 \mathrm{~kg} / \mathrm{m}^{3}, \gamma_{l v}=72.75 \mathrm{mN} / \mathrm{m}$, and $\mu=1.0087 \mathrm{mPa} \mathrm{s}$ (Ref. 16).

kinetic energy is the result of the competition between the viscous energy and surface energy, which can be described by the Ohnesorge number $\mathrm{On}=\mu / \sqrt{\rho \gamma_{l v} r} .{ }^{14}$ For droplets of small size (submicron), the Ohnesorge number is larger than the critical value $0.1,{ }^{15}$ which indicates that the viscous dissipation dominates during the coalescence and, consequently, there is no energy available for the self-propelled behavior. Interestingly, this corresponds to the "immobile coalescence" reported by Boreyko and Chen. ${ }^{6}$

Once the self-propelled phenomenon emerges, the coalescence-induced velocity $V$ of the coalescent droplet can be found from Eq. (9) as

$$
V=\sqrt{\frac{\gamma_{l v}}{\rho r}}\left[\frac{3}{8} C(f, \theta)-24 \frac{\mu}{\sqrt{\rho \gamma_{l v} r}}\right]^{1 / 2},
$$

where $C(f, \theta)$ is the term in the curly brackets of Eq. (9).

The water properties at $20{ }^{\circ} \mathrm{C}$ are used for the following calculation, such as $\rho=998.23 \mathrm{~kg} / \mathrm{m}^{3}, \gamma_{l v}=72.75 \mathrm{mN} / \mathrm{m}$, and $\mu=1.0087 \mathrm{mPa} \mathrm{s} .{ }^{16}$ Without loss of generality, the other parameters are assumed to be $\theta=170^{\circ}, \theta_{Y}=160^{\circ}, r_{f W}=1.5$, $f_{W}=1.0, r_{f C}=1.0$, and $f_{C}=0.5$. Moreover, the choice of these parameters has only a limited effect on the final results and does not change the trend observed from the analysis. Using these parameters, one can calculate the coalescence-induced velocity of water droplets. Figure 2 shows the dependence of the coalescence-induced velocity on the radius of water droplet. Obviously, the self-propelled behavior is not present for droplets of small size. This is due to the larger Ohnesorge number than the critical value 0.1 . For water droplets of large size, the coalescence-induced velocity first increases with the droplet radius to a maximum velocity and then decreases with the droplet radius. It should be noted that our analysis is based on the assumption that the gravity can be neglected. For droplets of larger size than the capillary length $l_{c}$, the variation of the gravitational-potential energy likely consumes the surface energy released upon the coalescence of droplets and then no coalescence-induced self-propelled behavior can be observed.

Our modeling analysis, with the viscous dissipation involved, captures several features of the experimental results 
reported by Boreyko and Chen, ${ }^{6}$ which includes: (i) the coalescence-induced velocity increases with the droplet size to a maximum and then decreases with the size, (ii) the maximum velocity can be found for the coalescence of droplets with the radius of about $50 \mu \mathrm{m}$, which is in accord with the experimental observation, and (iii) although the theoretical velocities are about two to three times larger than those measured in Boreyko and Chen's experiments, they are on the same order of magnitude.

In summary, this work has attempted to emphasize the importance of the viscous dissipation in examining the selfpropelled behavior during the coalescence of droplets over a superhydrophobic rough surface. The theoretical analysis reveals that the self-propelled behavior can only occur for the coalescence of droplets with the radius ranging from several microns to a few millimeters, in which the surface energy dominates compared with the viscous dissipation and the gravitational-potential energy. In particular, the study has shown that an equilibrium approach involving the conservation of energy can lead to a prediction of the coalescenceinduced velocity for the coalescence of two quasistationary liquid droplets of the same size. The coalescence-induced velocity is a function of the droplet size, which is consistent with the experimental results. By modifying the surface properties of liquid droplets through chemical treatment and controlling the droplet size, one can use the coalescenceinduced self-propelled behavior for the applications in selfcleaning, microfluidics, and lab-on-a-chip devices.
This work is jointly supported by the National Natural Science Foundation of China (NSFC, Grant Nos. 60936001 and 11072244), the National Basic Research Program of China (973 Program, Grant No. 2007CB310500), and Opening Fund of State Key Laboratory of Nonlinear Mechanics.

${ }^{1}$ F. Brochard-Wyart, in Soft Matter Physics, edited by M. Daoud and C. E. Williams (Springer, New York, 1999).

${ }^{2}$ R. Wenzel, Ind. Eng. Chem. 28, 988 (1936).

${ }^{3}$ A. Cassie and S. Baxter, Trans. Faraday Soc. 40, 546 (1944).

${ }^{4}$ J. B. Boreyko and C. H. Chen, Phys. Rev. Lett. 103, 174502 (2009).

${ }^{5}$ T. N. Krupenkin, J. A. Taylor, E. N. Wang, P. Kolodner, M. Hodes, and T. R. Salamon, Langmuir 23, 9128 (2007).

${ }^{6}$ J. B. Boreyko and C. H. Chen, Phys. Rev. Lett. 103, 184501 (2009).

${ }^{7}$ C. H. Chen, Q. J. Cai, C. L. Tsai, C. L. Chen, G. Y. Xiong, Y. Yu, and Z. F. Ren, Appl. Phys. Lett. 90, 173108 (2007).

${ }^{8}$ C. Dorrer and J. Ruhe, Adv. Mater. 20, 159 (2008).

${ }^{9}$ B. Mockenhaupt, H.-J. Ensikat, M. Spaeth, and W. Barthlott, Langmuir 24, 13591 (2008)

${ }^{10}$ J. N. Israelachvili, Intermolecular and Surface Forces (Academic, San Diego, 1992).

${ }^{11}$ A. Marmur, Langmuir 19, 8343 (2003).

${ }^{12}$ S. Chandra and C. T. Avedisian, Proc. R. Soc. London, Ser. A 432, 13 (1991)

${ }^{13}$ S. T. Thoroddsen and K. Takehara, Phys. Fluids 12, 1265 (2000).

${ }^{14}$ F. C. Wang, J. T. Feng, and Y. P. Zhao, J. Colloid Interface Sci. 326, 196 (2008).

${ }^{15}$ R. Q. Duan, S. Koshizuka, and Y. Oka, Nucl. Eng. Des. 225, 37 (2003).

${ }^{16}$ CRC Handbook of Physics and Chemistry, 63rd ed., edited by R. C. Weast and M. J. Astle (CRC, Boca Raton, 1982). 